Case report

\title{
Borderline personality features possibly related to cingulate and orbitofrontal cortices dysfunction due to schizencephaly
}

\author{
Felipe Filardi da Rocha ${ }^{a}$, Leandro Malloy-Diniz ${ }^{\mathrm{b}}$, Karla Cristhina Alves de Sousa ${ }^{\mathrm{c}}$, \\ Hugo Alejandro Cano Prais ${ }^{c}$, Humberto Correa ${ }^{c}$, Antonio Lucio Teixeira ${ }^{d}$,* \\ ${ }^{a}$ Department of Pharmacology, Institute of Biological Sciences, Federal University of Minas Gerais, Belo Horizonte, Brazil \\ b Neuropsychology and Neurosciences Research Group, Health Sciences School, FUMEC University, Belo Horizonte, Minas Gerais, Brazil \\ ${ }^{c}$ Department of Mental Health, School of Medicine, Federal University of Minas Gerais, Belo Horizonte, Brazil \\ ${ }^{\mathrm{d}}$ Department of Internal Medicine, School of Medicine, Federal University of Minas Gerais, Belo Horizonte, Brazil
}

Received 28 August 2007; received in revised form 12 November 2007; accepted 13 December 2007

\begin{abstract}
Prefrontal cortex dysfunction has been associated with a series of behavioral symptoms, such as impulsivity and affective instability, which are the defining features of several personality disorders, notably, borderline personality disorder. We report on a 27-year-old patient with schizencephaly in the right frontal lobe (cingulate cortex lesion and secondary orbitofrontal cortex dysfunction) presenting with prominent borderline features and compromise of executive functions, decision-making and attention. We hypothesize that the personality disorder of our patient could be related to cingulate cortex lesion and secondary orbitofrontal cortex dysfunction associated with schizencephaly.

(C) 2007 Elsevier B.V. All rights reserved.
\end{abstract}

Keywords: Schizencephaly; Impulsivity; Borderline personality; Orbitofrontal cortex; Cingulate cortex

\section{Introduction}

Schizencephaly (SCHZ) is a rare congenital disorder of cerebral cortical development. SCHZ is characterized by abnormal slits or clefts in the brain's cerebral hemispheres. It is a form of porencephaly in which there is a cyst or a cavity in the cerebral hemispheres. An anomaly of neuronal migration during the second trimester of gestation period is considered the most likely explanation for schizencephaly [1].

Lesions of the prefrontal region, mainly orbital and medial prefrontal cortices, have been associated with a series of behavioral symptoms, such as impulsivity and affective instability, which are the defining features of several personality disorders, notably, borderline personality disorder (BPD) [2]. Although neuroimaging studies demonstrated differences in the prefrontal cortex of patients with BPD compared to

\footnotetext{
* Corresponding author at: Departamento de Clínica Médica, Faculdade de Medicina, Universidade Federal de Minas Gerais, Av. Alfredo Balena, 190 Santa Efigênia, Belo Horizonte 30130-100, Brazil. Tel.: +55 21 34992651; fax: +552134992651 .

E-mail address: altexr@gmail.com (A.L. Teixeira).
}

healthy subjects [2-4], there is no published report correlating borderline features and SCHZ.

We describe a patient with SCHZ in the right frontal lobe presenting with a personality disorder marked by prominent borderline features.

\section{Case report}

A 27-year-old man was admitted to our psychiatric ward following a judicial order due to the involvement in episodes of aggression and attempted robbery.

Behavioral problems of the patient were noticed for the first time when he was 8 years old. At this age, he started recurrent fights with fellow pupils. During adolescence, he got involved in frequent episodes of exihibitionist behavior, substance abuse (i.e. sporadic marijuana and alcohol consumption) and displays of temper and aggression directed at his family members. Besides impulsive behavior, he has also presented with affective instability, a persistently unstable sense of self and a pattern of unstable and intense interpersonal relationships. 
Two structured clinical interviews, Mini International Neuropsychiatric Interview (M.I.N.I.)-Plus and Structured Clinical Interview for DSM Personality Disorders (SCID)II, were used to perform the diagnosis of DSM-IV Axis-I and Axis-II disorders, respectively. Psychoactive drug abuse (marijuana and alcohol) was identified but no other Axis-I disorder, including bipolar disorder, was noticed. Based on the patient's long-term history of impulsivity, affective liability and unstable sense of self and relationships, borderline features could be easily identified. Other borderline features, including transient paranoid ideation, feeling of emptiness or fear of abandonment, were not evidenced.

Careful laboratory investigation, including blood count, creatine kinase level, electrolytes, renal, liver and thyroid function tests, serology for syphilis and HIV, was undertaken and all exams were unrevealing. Brain magnetic resonance imaging (MRI) showed unilateral SCHZ of the closed lip variety (type 1) with clefts located in the right frontal lobe (Fig. 1A and B).

The patient was also submitted to a comprehensive neuropsychological assessment of intelligence (Wechsler adult intelligence scale, third edition; WAIS-III) [5], attention (omission errors on Conners' continuous performance test II; CPT-II) [6], motor impulsiveness (commission errors on CPT-II) [6], decision-making/cognitive impulsiveness (Iowa Gambling Task; IGT) [7], constructional ability (copy of the Rey Osterreich complex figure; SPREEN \& STRAUSS, 1998), visual memory (retrieval of the Rey Osterreich complex figure), verbal learning and memory (Rey auditory verbal learning test; RAVLT) [8], and language (verbal IQ and verbal comprehension indexes of WAIS-III and word fluency) $[5,8]$.

His performance on the neuropsychological tests was compared to the performance of asymptomatic male controls matched for age (mean \pm S.D., years; $28.2 \pm 1.4$ ) and years of formal education (mean \pm S.D., years; $7.3 \pm 1.2$ ). When the results of the patient differed two standard deviations above or bellow the mean value of control group they were considered altered.

He presented with normal intellectual level, although his performance was worse than in asymptomatic controls in the performance scale IQ, working memory and speed processing indexes of the WAIS-III (Table 1). There was no impairment of memory, language and visuoconstructional abilities. He committed several commission and omission errors on CPT-II indicating compromise of attention and motor impulsivity, respectively. He also was more susceptible to effects of proactive interference on learning process. On IGT, he made more non-profitable choices suggesting deficits in decisionmaking.

The result of his neuropsychological assessment is in line with the clinical symptoms presented by the patient, notably impulsivity. As the patient had SCHZ, he could not be diagnosed as BPD. According to DSM-IV diagnostic criteria, BPD can be diagnosed only in the absence of medical or neurological conditions that could explain psychiatric symp-
(A)

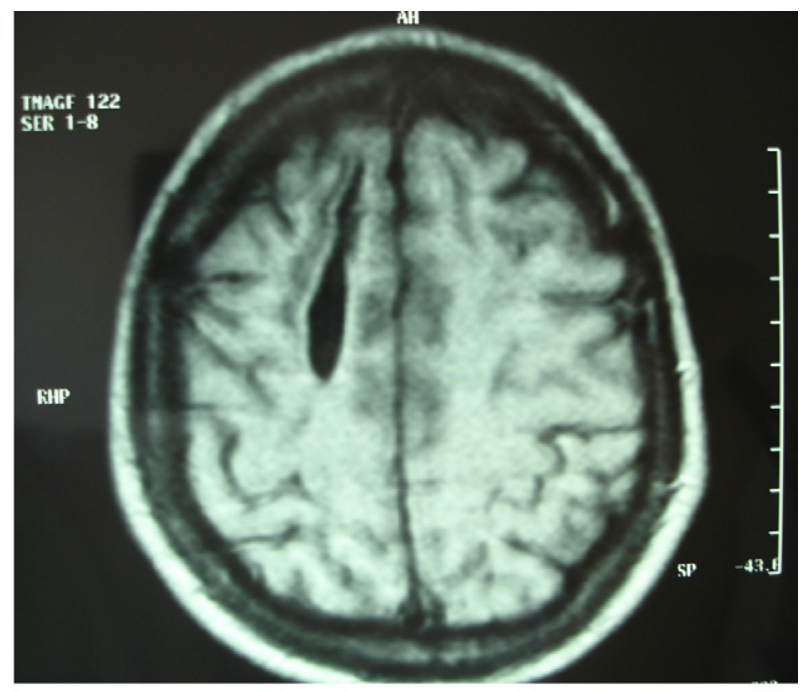

(B)



Fig. 1. T1-weighted magnetic resonance imaging showing unilateral schizencephaly (type 1) in the right frontal lobe: (A) axial image demonstrates a pore extending from the medial frontal cortex deep into the white matter to the level of lateral ventricles and (B) sagittal image shows the compromise of the cingulate gyrus.

toms. It is worth mentioning that the patient also displayed antisocial behaviors.

Under lithium carbonate $(1200 \mathrm{mg} / \mathrm{day})$ and pimozide $(8 \mathrm{mg} /$ day $)$ treatment there was improvement in his antisocial behavior. Nevertheless his impulse behavior remained unchanged as evidenced by the persistence of rage outbursts.

\section{Discussion}

The existence of a potential neurobiological basis for BPD is suggested by possible genetic susceptibility, serotonergic dysfunction, neuropsychological deficits, notably executive dysfunction and decision-making impairment, structural and functional brain abnormalities $[2,4,9]$. 
Table 1

Comparison of neuropsychological performance of the patient and ten asymptomatic male controls of approximately age (from 25 through 30 years old) and educational level (from 6 through 8 years of formal education)

\begin{tabular}{|c|c|c|}
\hline Test & Patient score & $\begin{array}{l}\text { Mean and } \\
\text { standard } \\
\text { deviation of } \\
\text { control group }\end{array}$ \\
\hline \multicolumn{3}{|l|}{ WAIS-III } \\
\hline Full scale IQ (FSIQ) & 90 & $102 \pm 6.4$ \\
\hline Verbal scale IQ (VSIQ) & 101 & $104 \pm 7.1$ \\
\hline Performance scale IQ (PSIQ) & 77 & $100 \pm 6.8$ \\
\hline Perceptual organization index (POI) & 89 & $100 \pm 7.9$ \\
\hline Verbal comprehension $(\mathrm{VCI})$ & 105 & $103 \pm 0.4$ \\
\hline Freedom of distractibility (FDI) & 79 & $101 \pm 7.3$ \\
\hline Working memory (WMI) & 73 & $104 \pm 8.2$ \\
\hline \multicolumn{3}{|l|}{ CPT-II } \\
\hline Omissions & 16 & $2.8 \pm 24$ \\
\hline Commissions & 28 & $8.1 \pm 5.2$ \\
\hline Hit reaction time & 426.64 & $368.5 \pm 62.3$ \\
\hline \multicolumn{3}{|l|}{ Rey's complex figure } \\
\hline Copy & 20 & $23.5 \pm 4.8$ \\
\hline Retrieval & 22 & $21.2 \pm 6.4$ \\
\hline \multicolumn{3}{|l|}{ RAVLT } \\
\hline A1-A5 & 42 & $51.6 \pm 7.4$ \\
\hline Proactive interference & 0.3 & $0.8 \pm 0.2$ \\
\hline Retroactive interference & 0.7 & $0.8 \pm 0.1$ \\
\hline Forget speed & 1 & $0.9 \pm 0.1$ \\
\hline Recognition memory & 12 & $11.8 \pm 2.7$ \\
\hline \multicolumn{3}{|l|}{ Word fluency-phonological } \\
\hline $\mathrm{F}$ & 13 & $13 \pm 3.8$ \\
\hline A & 10 & $9.9 \pm 4.1$ \\
\hline $\mathrm{S}$ & 12 & $13.7 \pm 4.5$ \\
\hline Word fluency—semantic (animals) & 14 & $18 \pm 3.3$ \\
\hline $\mathrm{F}$ (perseverative errors) & 2 & $0.8 \pm 1.1$ \\
\hline A (perseverative errors) & 1 & $0.7 \pm 0.9$ \\
\hline $\mathrm{S}$ (perseverative errors) & 1 & $0.9 \pm 0.6$ \\
\hline $\begin{array}{l}\text { Word fluency—semantic } \\
\quad \text { (animals-perseverative errors) }\end{array}$ & 1 & $1.1 \pm 1.3$ \\
\hline \multicolumn{3}{|l|}{ Iowa gambling task } \\
\hline CD-AB (first block of 20 choices) & 0 & $-1.2 \pm 5.3$ \\
\hline $\mathrm{CD}-\mathrm{AB}$ (second block of 20 choices) & 0 & $1.5 \pm 4.1$ \\
\hline CD-AB (third block of 20 choices) & -2 & $4.3 \pm 8.1$ \\
\hline $\mathrm{CD}-\mathrm{AB}$ (fourth block of 20 choices) & -16 & $6.4 \pm 5.9$ \\
\hline $\mathrm{CD}-\mathrm{AB}$ (fifth block of 20 choices) & -4 & $7.2 \pm 6.4$ \\
\hline
\end{tabular}

Cingulate cortex has been shown to subserve an array of functions, including emotional and attentional processing, as it is connected with amygdala, orbital and medial prefrontal cortices. Conversely orbital and medial prefrontal cortices are highly interconnected with different subcortical regions forming cortical-subcortical circuits. Based on lesion studies, it was suggested that structural damage to these circuits was related with disinhibition and changes of affect [10]. These clinical features are characteristic of disruptive behavior marked by outbursts of irritability and aggression. In line with this, some studies have even reported that cingulate cortex and adjacent regions, especially orbitofrontal cortex, which normally exert an inhibitory influence on behavior, are dysfunctional in BPD patients [2,3].
Thus we hypothesize that some features of the personality of our patient which resembles BPD, i.e. impulsivity and affect instability, could be related to cingulate cortex lesion and secondary orbitofrontal cortex dysfunction associated with SCHZ. Anti-social behavior presented by the patient could also be explained by an orbitofrontal dysfunction [10]. Interestingly, there is a high comorbidity between anti-social personality disorder and BPD [11].

The compromise of executive functions, decision-making and attention in the present case is compatible with dysfunction of different prefrontal cortex circuits. As cingulate gyrus is interconnected with prefrontal cortex and it is altered in the patient, these circuits were probably secondarily affected.

The evaluation of motor and cognitive impulsiveness by CPT-II (commission errors) and IGT, respectively, showed that the patient had impaired inhibitory control and inability to suppress immediate reward that could lead to subsequent loss. This pattern of poor decision-making was also observed in patients of orbitofrontal lesions [6] and with several psychiatric disorders including BPD [9,12]. Attention deficits are associated with prefrontal impairment, mainly when posterior cingulate gyrus is involved [13], and they have also been related to BPD [14].

Interestingly the great difference between verbal scale IQ and performance scale IQ indexes of WAIS-III is frequently found in BPD [5], in some psychiatric disorders presenting with executive dysfunction, such as schizophrenia [15] and right frontal pathologies [16].

It is worth to mention that pharmacologic treatment of BPD remains limited and restricted to symptomatic attenuation. In the present case, lithium and pimozide were chosen due to the presence of significant impulsive behavior and affective instability. The poor symptomatic control in our patient corroborates the hypothesis of prefrontal system lesion and/or dysfunction.

In conclusion structural and/or functional compromise of discrete prefrontal and cingulate areas may lead to borderline symptoms, mainly affective instability and impulsivity. This may also suggest that other borderline symptoms, such as fear of abandonment and paranoia, could be related to other brain circuits dysfunction.

\section{Acknowledgement}

This work was partly funded by grants from Conselho Nacional de Desenvolvimento Científico e Tecnológico (CNPq, Brazil).

\section{References}

[1] Liang JS, Lee WT, Peng SS, Yu TW, Shen YZ. Schizencephaly: correlation between clinical and neuroimaging features. Acta Paediatr Taiwan 2002;43:208-13. 
[2] Berlin HA, Rolls ET, Iversen SD. Borderline personality disorder, impulsivity, and the orbitofrontal cortex. Am J Psychiatry 2005;162:2360-73.

[3] Hazlett EA, New AS, Newmark R, Haznedar MM, Lo JN, Speiser LJ, et al. Reduced anterior and posterior cingulate gray matter in borderline personality disorder. Biol Psychiatry 2005;58:614-23.

[4] Schmahl C, Bremner JD. Neuroimaging in borderline personality disorder. J Psychiatr Res 2006;40:419-27.

[5] Wechsler D. WAIS-III: administration and scoring manual. San Antonio: The Psychological Corporation; 1997.

[6] Conners CK. Conners' continuous performance test manual. 2nd ed. Toronto, Canada: Multi-Health Systems; 2000.

[7] Bechara A, Damasio A, Damasio H, Anderson SW. Insensitivity to future consequences following damage to human prefrontal cortex. Cognition 1994;50:7-12.

[8] Strauss E, Sherman E, Spreen OA. Compendium of Neuropsychological Tests. 3rd ed. New York: Oxford University Press; 2006.

[9] LeGris J, van Reekum R. The neuropsychological correlates of borderline personality disorder and suicidal behaviour. Can J Psychiatry 2006;51:131-42.
[10] Bechara A, Damasio H, Damasio AR. Emotion, decision making and the orbitofrontal cortex. Cereb Cortex 2000;10:295-307.

[11] Goodman G, Hull JW, Clarkin JF, Yeomans FE. Childhood antisocial behaviors as predictors of psychotic symptoms and DSM-III-R borderline criteria among inpatients with borderline personality disorder. J Personal Disord 1999;13:35-46.

[12] Bazanis E, Rogers RD, Dowson JH, Taylor P, Meux C, Staley C, et al. Neurocognitive deficits in decision-making and planning of patients with DSM-III-R borderline personality disorder. Psychol Med 2002;32:1395-405.

[13] Rueckert L, Grafman J. Sustained attention deficits in patients with right frontal lesions. Neuropsychologia 1996;34:953-63.

[14] Posner MI, Rothbart MK, Vizueta N, Levy KN, Evans DE, Thomas $\mathrm{KM}$, et al. Attentional mechanisms of borderline personality disorder. Proc Natl Acad Sci USA 2002;99:16366-70.

[15] Iverson GL, Woodward TS, Green P. Base rates of WAIS-R VIQPIQ differences in 1593 psychiatric inpatients. J Clin Psychol 2001;57:1579-87.

[16] Boone KB, Miller BL, Lee A, Berman N, Sherman D, Stuss DT. Neuropsychological patterns in right versus left frontotemporal dementia. J Int Neuropsychol Soc 1999;5:616-22. 